\title{
Post Kala-Azar Dermal Leishmaniasis
}

National Cancer Institute

\section{Source}

National Cancer Institute. Post Kala-Azar Dermal Leishmaniasis. NCI Thesaurus. Code C34936.

A cutaneous form of leishmaniasis which sometimes occurs after visceral leishmaniasis treatment. It is characterized by hypo-pigmented macules, papules, plaques, nodules, or facial erythema; and is considered to be a durable infection reservoir for visceral leishmaniasis. 